ESAIM: M2AN

M2AN, Vol. 41, No 1, 2007, pp. 111-127

DOI: $10.1051 / \mathrm{m} 2 \mathrm{an}: 2007012$
ESAIM: Mathematical Modelling and Numerical Analysis

www.edpsciences.org/m2an

\title{
A MULTISCALE CORRECTION METHOD FOR LOCAL SINGULAR PERTURBATIONS OF THE BOUNDARY*
}

\author{
MARC DAmbrine ${ }^{1}$ AND GRÉGory ViaL ${ }^{2}$
}

\begin{abstract}
In this work, we consider singular perturbations of the boundary of a smooth domain. We describe the asymptotic behavior of the solution $u_{\varepsilon}$ of a second order elliptic equation posed in the perturbed domain with respect to the size parameter $\varepsilon$ of the deformation. We are also interested in the variations of the energy functional. We propose a numerical method for the approximation of $u_{\varepsilon}$ based on a multiscale superposition of the unperturbed solution $u_{0}$ and a profile defined in a model domain. We conclude with numerical results.
\end{abstract}

Mathematics Subject Classification. 35B25, 35B40, 35J25, 49Q10, 65N30.

Received: June 12, 2006. Revised: November 8 and 15, 2006.

\section{INTRODUCTION}

Various physical situations involve materials with a two-scale structure. From the macroscopic point of view, the considered body can usually be modeled by a smooth domain of $\mathbb{R}^{2}$ or $\mathbb{R}^{3}$, but this does not take into account the microscopic design of the material. We are specially interested in small inhomogeneities or cavities located on the border of the body. If they are arranged within a periodical network, homogenization techniques (see [1], for example) apply and a macroscopic model is valid, provided the characteristic properties of the material are modified accordingly. Such methods do not hold for local inhomogeneities, which are in the applications usually either omitted (for the smallest ones) or integrated into the macroscopic domain. Naturally, the numerical approximation of such problems requires a severe mesh refinement near the perturbation, which sometimes prevents from taking them into account in the computations.

In this paper, we deal with an elliptic partial differential equation in a domain with a small local boundary perturbation. We give the complete asymptotic expansion of its solution with respect to the size of the perturbing pattern, derive the variation of the associated energy (topological derivative) and propose a numerical method for the approximation of its solution based on the theoretical study.

Let us describe the geometrical setting we shall work within: $\Omega_{0}$ is an open bounded subset of $\mathbb{R}^{2}$ with smooth boundary containing the origin $O$. We assume, for simplicity in a first time, that the boundary $\partial \Omega_{0}$ coincides with a straight line near the origin, precisely for $|x|<r^{*}$. We will also deal in this work with smooth curved boundaries. On the other hand, $\mathbf{H}_{\infty}$ denotes an infinite domain of $\mathbb{R}^{2}$, which coincides with the upper

\footnotetext{
Keywords and phrases. Multiscale asymptotic analysis, shape optimization, patch of elements.

* This work has been partially supported by the ANR (project MACADAM $N^{\circ}$ JCJC06-139561).

1 LMAC, Université de Technologie de Compiègne, France.

2 IRMAR, Antenne de Bretagne de l'ENS Cachan, France. gvial@bretagne.ens-cachan.fr
} 

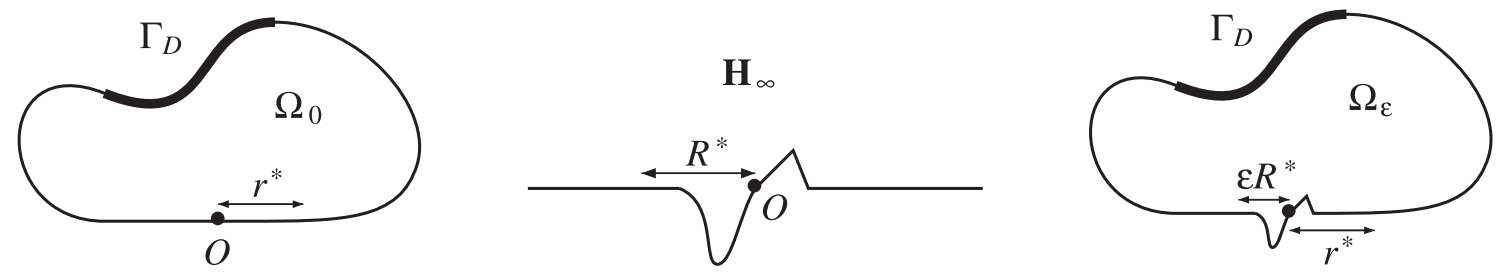

Figure 1. The original and pertubed domains.

half-plane at infinity, precisely for $|x|>R^{*}$. The perturbed domain $\Omega_{\varepsilon}$ is defined for small $\varepsilon$ by (see Fig. 1)

$$
\Omega_{\varepsilon}=\left\{x \in \Omega_{0} ;|x|>\varepsilon R^{*}\right\} \cup\left\{x \in \varepsilon \mathbf{H}_{\infty} ;|x|<r^{*}\right\} .
$$

Let us mention that we make no assumption of inclusion of the perturbed domain into the original one (or conversely). We will extend this framework to some curved smooth situations.

We define $u_{\varepsilon}$ as the solution in $\mathrm{H}^{1}\left(\Omega_{\varepsilon}\right)$ of the equation $-\Delta u_{\varepsilon}=f$ in $\Omega_{\varepsilon}$, where $f$ is some function in $\mathrm{L}^{2}\left(\Omega_{0}\right)$ vanishing in a neighborhood of the origin. We consider Dirichlet boundary conditions on $\Gamma_{D} \subset \partial \Omega_{\varepsilon}$ (which does not reach the origin) and Neumann boundary conditions elsewhere (other types of boundary conditions can also be treated). The asymptotic analysis of similar problems have been investigated by several authors in various special cases, see $[8,10,15,16]$. We adopt here the point of view of multiscale asymptotic expansions rather than the method of matching of asymptotic expansions - for a comparison of the two approaches, see [18]. It appears that the solution $u_{\varepsilon}$ can be approximated at first order by a superposition of the unperturbed solution $u_{0}$ and a profile, via cut-off functions in slow and rapid variables:

$$
u_{\varepsilon}=\zeta\left(\frac{x}{\varepsilon}\right) u_{0}(x)+\chi(x) W^{1}\left(\frac{x}{\varepsilon}\right)+\mathcal{O}_{\mathrm{H}^{1}\left(\Omega_{\varepsilon}\right)}\left(\varepsilon^{2}\right) .
$$

The cut-off functions $\zeta$ and $\chi$ are chosen smooth, radial, and satisfying

- the function $\zeta(x)$ equals 1 for $|x|>R^{*}$, and vanishes for $|x|<R^{*} / 2$;

- the function $\chi(x)$ equals 1 for $|x|<r^{*} / 2$ and vanishes for $|x|>r^{*}$.

The profile $W^{1}$ is defined as the solution in the domain $\mathbf{H}_{\infty}$ of a homogeneous model problem. In the expansion (2), the term $u_{0}$ only contributes away from the origin and the information concerning the perturbing pattern is carried by the profile. These two contributions interact in the transition zone through the cut-off functions.

We can base a numerical approach for the approximation of $u_{\varepsilon}$ on formula (2). Indeed, the computation of the term $u_{0}$ does not involve the perturbation and may therefore be done on a coarse mesh of $\Omega_{0}$. If we have a suitable approximation of the profile $W^{1}$, the superposition formula (2) gives a numerical solution for $u_{\varepsilon}$. The cut-off functions are handled in the practical process by means of patch of elements.

Moreover, expression (2) allows to compute the topological derivative - see $[12,13,17]$ - of the energy $j(\varepsilon)$ :

$$
j(\varepsilon):=-\frac{1}{2} \int_{\Omega_{\varepsilon}}\left|\nabla u_{\varepsilon}\right|^{2}=j(0)+\varepsilon^{2}\left|\nabla u_{0}(0)\right|^{2} \mathcal{A}_{\mathbf{H}_{\infty}}+\mathcal{o}\left(\varepsilon^{2}\right),
$$

where the real number $\mathcal{A}_{\mathbf{H}_{\infty}}$ only depends on the geometry of $\mathbf{H}_{\infty}$.

The paper is divided as follows. In a first section, we give the full asymptotic expansion of the state function in the case of a straight boundary near the origin, this is based on a multiscale asymptotic method. We extend then these results to a curved case. As far as we know, such a curved geometry with a self-similar perturbation has not been considered so far. Next, we derive the leading terms in the asymptotical description of the energy functional. The last part is devoted to the numerical method using patch of elements near 
the perturbation: a numerical validation of our theoretical results is given in the studied model case of the Laplace equation. We conclude the paper with comments on a possible application of the methods and technics developped in the present paper to mechanical engineering.

\section{Asymptotic expansion of the state FunCtion}

We consider the solution $u_{\varepsilon}$ of the following problem, posed in the geometry described by Figure 1:

$$
\left\{\begin{aligned}
-\Delta u_{\varepsilon} & =f \text { in } \Omega_{\varepsilon} \\
u_{\varepsilon} & =0 \text { on } \Gamma_{D} \\
\partial_{\mathbf{n}} u_{\varepsilon} & =0 \text { on } \partial \Omega_{\varepsilon} \backslash \Gamma_{D}
\end{aligned}\right.
$$

The technique we use to build an asymptotic expansion of $u_{\varepsilon}$ into powers of the small parameter $\varepsilon$ is adapted from the multi-scale approach of [18].

We first write the Taylor expansion at a target precision $K$ of the limit term $u_{0}$ at point $x=0$ (thanks to standard elliptic regularity, $u_{0}$ is a smooth function up to the boundary):

$$
u_{0}(x)=\chi(x) \sum_{k=0}^{K} u^{k}(x)+R_{K}(x)=\chi(x) T_{K}(x)+R_{K}(x),
$$

the first terms of the Taylor polynomial $T_{K}$ being given by $u^{0}(x)=u_{0}(0), u^{1}(x)=\left|\nabla u_{0}(0)\right| x_{1}$ (more generally $u^{k}$ is a homogeneous polynomial of total degree $k$ ). The limit term $u_{0}$ is not necessarily defined in the whole domain $\Omega_{\varepsilon}$, but its Taylor part may be extended to $\Omega_{\varepsilon}$. For this reason, a better start is given by the truncated function

$$
\tilde{u}_{0}(x)=\chi(x) T_{K}(x)+\zeta\left(\frac{x}{\varepsilon}\right) R_{K}(x) \in \mathrm{H}^{1}\left(\Omega_{\varepsilon}\right) .
$$

The difference between $u_{0}$ and $\tilde{u}_{0}$ is small since the remainder $R_{K}$ is flat in the cut-off region. Let us denote by $r_{\varepsilon}^{0}$ the difference between $u_{\varepsilon}$ and $\tilde{u}_{0}$, it naturally satisfies the following problem

$$
\left\{\begin{aligned}
-\Delta r_{\varepsilon}^{0} & =\varphi_{\varepsilon}^{0} & & \text { in } \Omega_{\varepsilon}, \\
r_{\varepsilon}^{0} & =0 & & \text { on } \Gamma_{D}, \\
\partial_{\mathbf{n}} r_{\varepsilon}^{0} & =-\chi(x) \partial_{\mathbf{n}} T_{K}+\psi_{\varepsilon}^{0} & & \text { on } \partial \Omega_{\varepsilon} \backslash \Gamma_{D},
\end{aligned}\right.
$$

where the data $\varphi_{\varepsilon}^{0}$ and $\psi_{\varepsilon}^{0}$ arise from the cut-off and are supported in the ring of size $\varepsilon$ defined as $\{x \in$ $\left.\Omega_{\varepsilon} ; \varepsilon R^{*} / 2<|x|<\varepsilon R^{*}\right\}$, they will contribute to the remainder since they are essentially of order $\varepsilon^{K}$. Thus, the principal defect in equation (8) comes from the normal derivative of the Taylor expansion of $u_{0}$, whose leading term reads

$$
-\chi(x)\left|\nabla u_{0}(0)\right| \partial_{\mathbf{n}} x_{1}=-\chi(x)\left|\nabla u_{0}(0)\right| n_{1}
$$

which does not vanish only on the boundary part of $\Omega_{\varepsilon}$ which corresponds to the perturbing pattern (the vector $\mathbf{n}=\left(n_{1}, n_{2}\right)$ stands for the unitary outer normal on $\left.\partial \Omega_{\varepsilon}\right)$. Following the ideas of $[4,5,18]$, we introduce the profile $V^{1}$ as the solution of the problem in the infinite domain $\mathbf{H}_{\infty}$ :

$$
\left\{\begin{aligned}
-\Delta V^{1} & =0 & & \text { in } \mathbf{H}_{\infty} \\
\partial_{\mathbf{n}} V^{1} & =-\left|\nabla u_{0}(0)\right| N_{1} & & \text { on } \partial \mathbf{H}_{\infty} \\
V^{1} & \rightarrow 0 & & \text { at infinity }
\end{aligned}\right.
$$

where $N_{1}$ denotes the first component of the unitary inner normal vector on $\partial \mathbf{H}_{\infty}$. The following lemma states the well-posedness of such a problem. 
Lemma 2.1. Problem (10) admits a unique weak solution $V^{1}$ in the variational space

$$
\left\{V ; \nabla V \in \mathrm{L}^{2}\left(\mathbf{H}_{\infty}\right) \quad \text { and } \quad \frac{V}{(1+|X|) \log (2+|X|)} \in \mathrm{L}^{2}\left(\mathbf{H}_{\infty}\right)\right\} .
$$

Furthermore, we have the following behaviors at infinity:

$$
V^{1}(X)=\mathcal{O}\left(|X|^{-1}\right) \quad \text { and } \quad \nabla V^{1}(X)=\mathcal{O}\left(|X|^{-2}\right) \quad \text { as }|X| \rightarrow \infty .
$$

The proof is given in [4]: existence and uniqueness in the variational space follows from a weighted Poincaré-like inequality, the behavior at infinity may be proven thanks to the tool of Mellin transform.

Using the profile $V^{1}$, we are able to write the beginning of the asymptotic expansion of $u_{\varepsilon}$ : we set

$$
r_{\varepsilon}^{1}=u_{\varepsilon}-\left[\tilde{u}_{0}+\chi(x) \varepsilon V^{1}\left(\frac{x}{\varepsilon}\right)\right] .
$$

By construction, this remainder satisfies

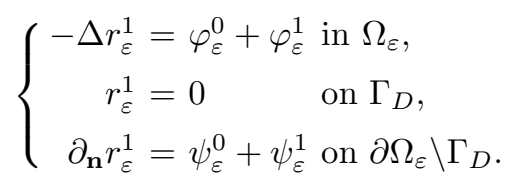

The function $\varphi_{\varepsilon}^{1}$ comes from the cut-off function $\chi$ :

$$
\varphi_{\varepsilon}^{1}=\Delta\left[\chi(\cdot) \varepsilon V^{1}(\dot{\bar{\varepsilon}})\right] .
$$

Note that in the Laplacian, only derivatives of $\chi$ are involved since $V^{1}$ is harmonic, and only $|x|>r^{*} / 2$ has to be considered in (15). The function $\psi_{\varepsilon}^{1}$ has its support inside the ball $|x|<\varepsilon R^{*}$ and is given by

$$
\psi_{\varepsilon}^{1}=-\chi(x) \partial_{\mathbf{n}} V^{1}\left(\frac{x}{\varepsilon}\right)-\chi(x) \partial_{\mathbf{n}} T_{K}=-\chi(x) \sum_{k=2}^{K} \partial_{\mathbf{n}} u^{k}(x)=\mathcal{O}_{\mathrm{L}^{2}\left(\Omega_{\varepsilon}\right)}\left(\varepsilon^{2}\right),
$$

since $V^{1}$ stands for the term corresponding to $k=1$ of the Taylor expansion (the constant term $u^{0}$ does not contribute to the normal derivative).

It is not straightforward to obtain a remainder estimate on $r_{\varepsilon}^{1}$ since the $\mathrm{L}^{2}$-norm of $\varphi_{\varepsilon}^{1}$ is only $\mathcal{O}(1)$. We need to build further terms to get the (optimal) estimate

$$
\left\|r_{\varepsilon}^{1}\right\|_{\mathrm{H}^{1}\left(\Omega_{\varepsilon}\right)}=\mathcal{O}\left(\varepsilon^{2}\right)
$$

The proof will follow from Theorem 2.2 below.

To continue the construction of the expansion, we need to take into account the next terms in the Taylor expansion of $u_{0}$ by new profiles, and add correctors for the cut-off. The technology used in $[4,5,18]$ can be extended, the main differences have been described just above for the first terms. Precisely, we get

Theorem 2.2. We assume that $f$ is an $\mathrm{L}^{2}$-function, with compact support inside $\Omega_{0}$. Then the solution $u_{\varepsilon}$ of (5) admits the following asymptotic expansion for $N<K$

$$
u_{\varepsilon}(x)=\tilde{u}_{0}(x)+\chi(x) \sum_{i=1}^{N} \varepsilon^{i} V^{i}\left(\frac{x}{\varepsilon}\right)+\sum_{i=2}^{N} \varepsilon^{i} w_{\varepsilon}^{i}(x)+\mathcal{O}_{\mathrm{H}^{1}\left(\Omega_{\varepsilon}\right)}\left(\varepsilon^{N+1}\right) .
$$

The term $\tilde{u}_{0}$ is defined by (7), the profile $V^{i}$ is a counterpart for the $i^{\text {th }}$ term $u^{i}$ of the Taylor expansion of $u_{0}$ - see $(20)$ - and $w_{\varepsilon}^{i}$ is a cut-off corrector satisfying $\left\|w_{\varepsilon}^{i}\right\|_{\mathrm{H}^{1}\left(\Omega_{\varepsilon}\right)}=\mathcal{O}(1)$. 
Proof of Theorem 2.2. We give a sketch of the proof for the complete asymptotic expansion. Supposing the expansion built until rank $N-1$, we set

$$
r_{\varepsilon}^{N}(x)=u_{\varepsilon}(x)-\tilde{u}_{0}(x)-\chi(x) \sum_{i=1}^{N-1} \varepsilon^{i} V^{i}\left(\frac{x}{\varepsilon}\right)-\sum_{i=2}^{N-1} \varepsilon^{i} w_{\varepsilon}^{i}(x),
$$

the remainder of order $N-1$. By definition, the profiles $V^{i}$ satisfies

$$
\left\{\begin{aligned}
-\Delta V^{i} & =0 & & \text { in } \mathbf{H}_{\infty} \\
\partial_{\mathbf{n}} V^{i} & =-\partial_{\mathbf{n}} u^{i} & & \text { on } \partial \mathbf{H}_{\infty} \\
V^{i} & \rightarrow 0 & & \text { at infinity }
\end{aligned}\right.
$$

(again, the datum is compactly supported and Lem. 2.1 ensures $^{1}$ existence and uniqueness of $V^{1}$ ).

Laplacian. By construction, the residual in $\Delta r_{\varepsilon}^{N}$ is corrected up to order $N-1$ by the $w_{\varepsilon}^{i}$. But the term $\Delta\left[\chi(x) \varepsilon^{N-1} V^{N-1}\left(\frac{x}{\varepsilon}\right)\right]$ is of order $\varepsilon^{N}$ in $\mathrm{L}^{\infty}\left(\Omega_{\varepsilon}\right)$ thanks to an estimate similar to (12). We define hence $w_{\varepsilon}^{N}$ as the solution in $\mathrm{H}^{1}\left(\Omega_{\varepsilon}\right)$ of

$$
-\Delta w_{\varepsilon}^{N}=-\Delta\left[\chi(x) \varepsilon^{N-1} V^{N-1}\left(\frac{x}{\varepsilon}\right)\right] \quad \text { with same boundary conditions as } u_{0} .
$$

Boundary conditions. The Dirichlet boundary condition on $\Gamma_{D}$ is fully satisfied by $r_{\varepsilon}^{N}$, but the Neumann boundary condition is not. Indeed, only the $N-1$ first Neumann-traces have been taken into account so far by the profiles $V^{i}$ : the leading term in $\partial_{\mathbf{n}} r_{\varepsilon}^{N}$ on $\partial \Omega_{\varepsilon} \backslash \Gamma_{D}$ is given by $-\partial_{\mathbf{n}} u^{N}(x)=-\varepsilon^{N} \partial_{\mathbf{n}} u^{N}\left(\frac{x}{\varepsilon}\right)$, by homogeneity. This naturally leads to the definition of $V^{N}$, according to (20).

Conclusion. The introduction of the terms $w^{N}$ and $V^{N}$ allows to define the remainder $r_{\varepsilon}^{N+1}$ of order $N$, which satisfies

- the Laplacian $-\Delta r_{\varepsilon}^{N+1}$ is small: precisely, its leading term is $\varepsilon^{N} \Delta\left[\chi(x) V^{N}\left(\frac{x}{\varepsilon}\right)\right]$, which is of order $\varepsilon^{N-1}$ in the $\mathrm{L}^{2}\left(\Omega_{\varepsilon}\right)$-norm;

- the Neumann boundary condition is satisfied up to a term in $\mathcal{O}_{\mathrm{L}^{2}\left(\partial \Omega_{\varepsilon}\right)}\left(\varepsilon^{N+1}\right)$ thanks to an estimate similar to (16).

Using an a priori estimate on problem (5) (independent on $\varepsilon$ ), we immediately get the rought estimate $r_{\varepsilon}^{N}=$ $\mathcal{O}_{\mathrm{H}^{1}\left(\Omega_{\varepsilon}\right)}\left(\varepsilon^{N-1}\right)$. To obtain the order $\varepsilon^{N+1}$, we simply write

$$
r_{\varepsilon}^{N}=r_{\varepsilon}^{N+2}+\chi(x) \varepsilon^{N+2} V^{N+2}\left(\frac{x}{\varepsilon}\right)+\varepsilon^{N+2} w_{\varepsilon}^{N+2}(x)+\chi(x) \varepsilon^{N+1} V^{N+1}\left(\frac{x}{\varepsilon}\right)+\varepsilon^{N+1} w_{\varepsilon}^{N+1}(x),
$$

yielding to the result from the estimates

$$
\chi(x) V^{k}\left(\frac{x}{\varepsilon}\right)=\mathcal{O}_{\mathrm{H}^{1}\left(\Omega_{\varepsilon}\right)}\left(\varepsilon^{k-1}\right) \quad \text { and } \quad w_{\varepsilon}^{k}=\mathcal{O}_{\mathrm{H}^{1}\left(\Omega_{\varepsilon}\right)}(1) .
$$

Remark 2.3. By a mere rearrangement of the terms, the expansion of $u_{\varepsilon}$ can read as follows

$$
u_{\varepsilon}=\zeta\left(\frac{x}{\varepsilon}\right) u_{0}(x)+\chi(x) \sum_{i=1}^{N} \varepsilon^{i} W^{i}\left(\frac{x}{\varepsilon}\right)+\sum_{i=2}^{N} \varepsilon^{i} \tilde{w}_{\varepsilon}^{i}(x)+\mathcal{O}_{\mathrm{H}^{1}\left(\Omega_{\varepsilon}\right)}\left(\varepsilon^{N+1}\right) .
$$

\footnotetext{
${ }^{1}$ Since Neumann conditions are considered, we have to make sure that the right hand-side of (20) meets the compatibility requirement. This is the case here: since $u_{0}$ is harmonic, it is also the case of the terms in its Taylor expansion.
} 

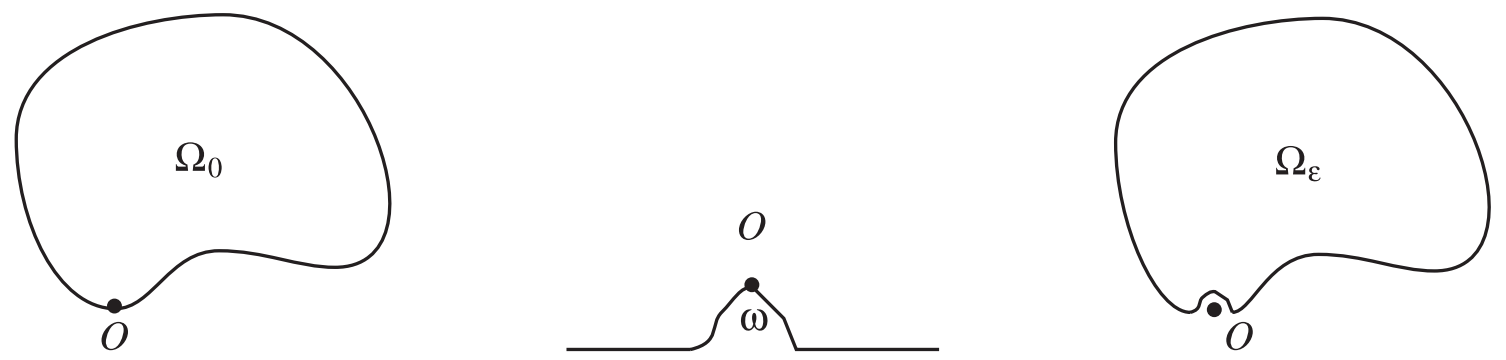

Figure 2. Domains in the case of locally convex curved boundary.

The new profiles $W^{i}$ are defined by $W^{i}(X)=V^{i}(X)+(1-\zeta(X)) u^{i}(X)$ and the $\tilde{w}_{\varepsilon}^{i}$ are new correctors. The advantage of this formulation is to involve $u_{0}$ itself, instead of $\tilde{u}_{0}$.

In the case of an cavity, i.e. $\Omega_{\varepsilon} \subset \Omega_{0}$, the function $\zeta$ can be chosen identically equal to 1 , and $W^{i}=V^{i}$.

Remark 2.4. We can deplore that the correcting terms $w_{\varepsilon}^{i}$ do depend on $\varepsilon$, though weakly since they are of order $\mathcal{O}(1)$ in the $\mathrm{H}^{1}\left(\Omega_{\varepsilon}\right)$-norm. It is possible to remove this feature from the asymptotic expansion by introducing correctors $z^{i}$ defined in the limit domain $\Omega_{0}$ (with same right-hand side), and using the cut-off function $\zeta$. Of course, the normal trace does no more vanish on the perturbed boundary and we have to take this into account in the definition of the profiles. The resulting expansion reads

$$
u_{\varepsilon}(x)=\tilde{u}_{0}(x)+\chi(x) \sum_{i=1}^{N} \varepsilon^{i} \tilde{V}^{i}\left(\frac{x}{\varepsilon}\right)+\zeta\left(\frac{x}{\varepsilon}\right) \sum_{i=2}^{N} \varepsilon^{i} z^{i}(x)+\mathcal{O}_{\mathrm{H}^{1}\left(\Omega_{\varepsilon}\right)}\left(\varepsilon^{N+1}\right) .
$$

or, with the previous remark,

$$
u_{\varepsilon}(x)=\zeta\left(\frac{x}{\varepsilon}\right) u_{0}(x)+\chi(x) \sum_{i=1}^{N} \varepsilon^{i} \tilde{W}^{i}\left(\frac{x}{\varepsilon}\right)+\zeta\left(\frac{x}{\varepsilon}\right) \sum_{i=2}^{N} \varepsilon^{i} \tilde{z}^{i}(x)+\mathcal{O}_{\mathrm{H}^{1}\left(\Omega_{\varepsilon}\right)}\left(\varepsilon^{N+1}\right)
$$

\section{Extension to some CURVED Boundaries}

In this section, for the lightness of the presentation, we consider the case of Dirichlet boundary conditions. Let $u_{\varepsilon}$ solve $-\Delta u=f$ in $\mathrm{H}_{0}^{1}\left(\Omega_{\varepsilon}\right)$ while $u_{0}$ solves the same equation in $\mathrm{H}_{0}^{1}\left(\Omega_{0}\right)$. We also restrict ourselves to the cavity case to avoid the need of $\tilde{u}_{0}$, and we make the assumption that the initial domain is convex in the neighborhood of $O$. The geometrical situation is illustrated in Figure 2.

This situation is not a mere extension of the flat one, considered previously. Indeed, if we rectify the boundary locally near $O$, the perturbation is not selfsimilar anymore in the new coordinates!

Following the analysis performed in [5], we introduce the profile $V_{d}^{1}$ as the solution of the problem in the infinite domain $\mathbf{H}_{\infty}$ :

$$
\left\{\begin{aligned}
-\Delta V_{d}^{1} & =0 & & \text { in } \mathbf{H}_{\infty}, \\
V_{d}^{1} & =-\left|\nabla u_{0}(0)\right| x_{2} & & \text { on } \partial \mathbf{H}_{\infty}, \\
V_{d}^{1} & \rightarrow 0 & & \text { at infinity, }
\end{aligned}\right.
$$

where $x_{2}$ denotes the second component of the position on $\partial \mathbf{H}_{\infty}$. As for the Neumann case, existence and uniqueness of such a profile follows from next lemma, similar to Lemma 2.1.

Lemma 3.1. Problem (10) admits a unique weak solution $V_{d}^{1}$ in the variational space

$$
\left\{V ; \nabla V \in \mathrm{L}^{2}\left(\mathbf{H}_{\infty}\right) \quad \text { and } \quad \frac{V}{1+|X|} \in \mathrm{L}^{2}\left(\mathbf{H}_{\infty}\right)\right\}
$$


Furthermore, there is a constant $C$ depending only $\mathbf{H}_{\infty}$ such that

$$
\left|V_{d}^{1}(X)\right| \leq \frac{C}{|X|} \quad \text { and } \quad\left|\nabla V_{d}^{1}(X)\right| \leq \frac{C}{|X|^{2}} \quad \text { when }|X| \rightarrow \infty \text {. }
$$

As in [5], we approximate $u_{\varepsilon}$ by $u_{0}+\chi V_{d}^{1}(\dot{\bar{\varepsilon}})$ and we set

$$
r_{\varepsilon}^{d}(x)=u_{\varepsilon}(x)-\left[u_{0}(x)+\chi(x) V_{d}^{1}\left(\frac{x}{\varepsilon}\right)\right] .
$$

This remainder solves

$$
\left\{\begin{array}{rlrl}
-\Delta r_{\varepsilon}^{d}(x) & =\Delta\left[\chi(x) \varepsilon V_{d}^{1}\left(\frac{x}{\varepsilon}\right)\right], & & \text { in } \Omega_{\varepsilon}, \\
r_{\varepsilon}^{d}(x) & =u_{0}(x)-\chi(x) \varepsilon V_{d}^{1}\left(\frac{x}{\varepsilon}\right) & \text { on } \partial \Omega_{\varepsilon} .
\end{array}\right.
$$

The difference with the flat case treated is the presence of non-vanishing boundary conditions on $\partial \Omega_{0} \cap \partial \Omega_{\varepsilon}$. The expansions obtained in [5] and in Section 2 were justified without taking into account the short range interaction between the profiles and the geometry of the initial domain $\Omega_{0}$. The flatness assumption of $\Omega_{0}$ around $O$ cancels the interaction between slow and rapid variable that we have to face in the curved case. Let us emphasize the fact that the approximation $u_{0}+\varepsilon \chi V_{d}^{1}(\dot{\bar{\varepsilon}})$ does not satisfy the homogeneous Dirichlet boundary conditions on $\partial \Omega_{0} \cap \partial \Omega_{\varepsilon}$. However, its trace almost vanishes.

Like in the previous section, the laplacian part is easy to handle and it holds:

$$
\left\|\Delta\left[\chi(x) \varepsilon V_{d}^{1}\left(\frac{x}{\varepsilon}\right)\right]\right\|_{L^{2}\left(\Omega_{\varepsilon}\right)} \leq C \varepsilon^{2} .
$$

We need to consider the boundary conditions on $\partial \Omega_{\varepsilon}$ in the two natural parts: on $\partial \Omega_{\varepsilon} \cap \Omega_{0}$, we immediately get $r_{\varepsilon}^{d}=u^{2}$, which is naturally of order $\varepsilon^{2}$ as a reminder of order 2 in a Taylor expansion. We have to prove that this estimate extends to $\partial \Omega_{0} \cap \partial \Omega_{\varepsilon}$. This proof turns out to be the most difficult part of the extension to curved boundaries. The leading idea of the analysis is a decomposition of profiles in terms of homogeneous functions, usually obtained from the Mellin transform, see [4,9]. Here, we only need the weak following statement.

Lemma 3.2. The profil $V_{d}^{1}$ can be written as the sum $\mathcal{V}_{d}^{1}+\mathcal{R}$ where $\mathcal{V}_{d}^{1}$ is a homogeneous function of degree -1 and the remainder $\mathcal{R}$ satisfies the following behavior at infinity: there is a constant $C$ depending only $\mathbf{H}_{\infty}$ such that

$$
|\mathcal{R}(X)| \leq \frac{C}{|X|^{2}} \quad \text { and } \quad|\nabla \mathcal{R}(X)| \leq \frac{C}{|X|^{3}} \quad \text { when }|X| \rightarrow \infty .
$$

Proof of Lemma 3.2. Fix $R>0$ large enough so that $\omega$ is included into the ball $B(O, R)$. Then, the trace of $V_{d}^{1}$ on the curve $\partial B(O, R) \cap \mathbf{H}_{\infty}$ is smooth and can be written as the sum of its Fourier series. Thanks to the boundary conditions, only the sine functions appear and one gets

$$
V_{d}^{1}(R, \theta)=a_{0}+\sum_{n \geq 1} a_{n} \sin n \theta
$$

Using Poisson's kernel, we then get that

$$
V_{d}^{1}(r, \theta)=a_{0}+\sum_{n \geq 1} a_{n} \frac{R^{n}}{r^{n}} \sin n \theta
$$

The behavior at infinity of $V_{d}^{1}$ prescribes $a_{0}=0$ and we set $\mathcal{V}_{d}^{1}(r, \theta)=a_{1} \frac{R}{r} \sin \theta$. Note that the dependency of the expression of $\mathcal{V}_{d}^{1}$ with respect to $R$ is fictitious thanks to its homogeneity. Setting $\mathcal{R}=V_{d}^{1}-\mathcal{V}_{d}^{1}$, leads to the stated result. 


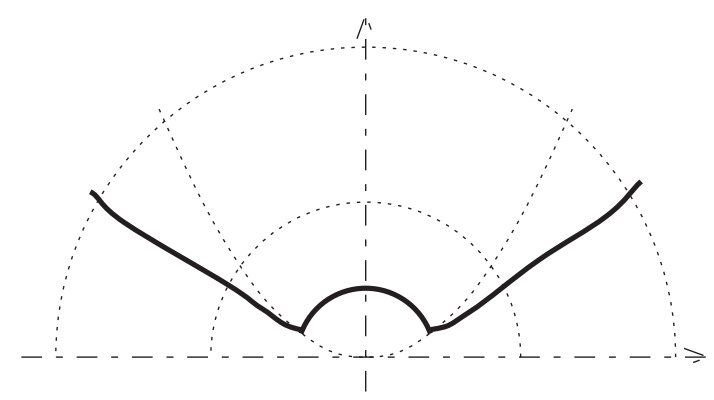

FiguRE 3. The geometrical setting of the cavity in the convex case.

Let us specify the geometry of $\partial \Omega_{0}$ around $O$. We assume $\partial \Omega_{0}$ to be $\mathcal{C}^{2}$ and fix the coordinate axis such that $\partial \Omega_{0}$ is the graph $x_{2}=h\left(x_{1}\right)$ of a function $h$ in the neighborhood of $O$ with $h(0)=h^{\prime}(0)=0$. Then, there exists a number $C>0$ and a radius $r>0$ such that for $x=\left(x_{1}, x_{2}\right) \in \partial \Omega_{0}$, it holds

$$
|x| \leq r \Rightarrow 0 \leq h\left(x_{1}\right) \leq C\left|x_{1}\right|^{2} \text { and }\left|h^{\prime}\left(x_{1}\right)\right| \leq C\left|x_{1}\right| ;
$$

this property is connected to the $\mathcal{C}^{2}$ regularity of $\partial \Omega_{0}$. We fix $r^{*}=r$ and choose $\varepsilon \ll r^{*}$ : the characteristic size of the perturbation is small with respect of the radius of curvature of $\partial \Omega_{0}$ at $O$. This assumption is a natural limitation of the method. The geometrical context is summed up in Figure 3.

We can now state the estimates on the boundary conditions. The term $\mathcal{V}_{d}^{1}$ is homogeneous of order -1 , therefore it is easy to check that $\left\|\mathcal{V}_{d}^{1}(\dot{\bar{\varepsilon}})\right\|_{\mathrm{H}^{1 / 2}\left(\partial \Omega_{\varepsilon}\right)}$ is of order $\varepsilon$. Thus, we focus on the remainder

$$
\tilde{r}_{\varepsilon}^{d}(x)=r_{\varepsilon}^{d}(x)+\varepsilon \chi \mathcal{V}_{d}^{1}\left(\frac{x}{\varepsilon}\right)=r_{\varepsilon}^{d}+\mathcal{O}_{\mathrm{H}^{1 / 2}\left(\partial \Omega_{\varepsilon}\right)}\left(\varepsilon^{2}\right) .
$$

Proposition 3.3. One has

$$
\left\|\tilde{r}_{\varepsilon}^{d}\right\|_{\mathrm{H}^{1 / 2}\left(\partial \Omega_{\varepsilon}\right)} \leq C \varepsilon^{2} .
$$

Proof of Proposition 3.3. For localization reasons (we will split the norm on subdomains of $\partial \Omega_{\varepsilon}$ ), we consider the $\mathrm{L}^{2}$ and $\mathrm{H}^{1}$ norms, the result on the $\mathrm{H}^{1 / 2}$ norm following by interpolation. Precisely, it is enough to prove

$$
\begin{aligned}
& \left\|\tilde{r}_{\varepsilon}^{d}\right\|_{\mathrm{L}^{2}\left(\partial \Omega_{\varepsilon}\right)} \leq C \varepsilon^{5 / 2}, \\
& \left\|\tilde{r}_{\varepsilon}^{d}\right\|_{\mathrm{H}^{1}\left(\partial \Omega_{\varepsilon}\right)} \leq C \varepsilon^{3 / 2} .
\end{aligned}
$$

Thanks to the assumption made on the truncation in slow variable, the only two areas to be considered are: (i) $\varepsilon \partial \omega$ the boundary of the cavity itself, and (ii) the part of $\partial \Omega_{\varepsilon} \backslash \varepsilon \partial \omega$ in the support of the cut-off $\chi$.

(i) On $\varepsilon \partial \omega, r_{\varepsilon}^{d}$ is by construction the remainder of order two in the Taylor expansion of $u_{\Omega_{0}}$. Therefore, it is smooth with an $\mathrm{L}^{\infty}$-norm of order $\varepsilon^{2}$ and there is a constant $C>0$ such that

$$
\int_{\varepsilon \partial \omega}\left(\tilde{r}_{\varepsilon}^{d}(s)\right)^{2} \mathrm{~d} s \leq C \varepsilon^{5}
$$

After one derivation, one looses one order and gets

$$
\int_{\varepsilon \partial \omega}\left(\nabla_{\tau} \tilde{r}_{\varepsilon}^{d}(s)\right)^{2} \mathrm{~d} s \leq C \varepsilon^{3},
$$

which corresponds to the stated estimates (34) and (35). 
(ii) For $x=\left(x_{1}, h\left(x_{1}\right)\right) \in \partial \Omega_{\varepsilon} \backslash \varepsilon \partial \omega$, the remainder $\tilde{r}_{\varepsilon}^{d}$ is

$$
\tilde{r}_{\varepsilon}^{d}\left(x_{1}, h\left(x_{1}\right)\right)=-\varepsilon \chi\left(x, h\left(x_{1}\right)\right) \mathcal{R}\left(\frac{\left(x, h\left(x_{1}\right)\right)}{\varepsilon}\right) .
$$

Now, for $x_{1} \in\left(-r^{*}, r^{*}\right)$, we take advantage of the homogeneous Dirichlet boundary conditions and write the remainder as an integral and make the change of variable $y=\varepsilon s$ :

$$
\varepsilon \mathcal{R}\left(\frac{\left(x, h\left(x_{1}\right)\right)}{\varepsilon}\right)=\varepsilon \int_{0}^{h\left(x_{1}\right) / \varepsilon} \partial_{2} \mathcal{R}\left(\frac{x}{\varepsilon}, s\right) \mathrm{d} s=\int_{0}^{h\left(x_{1}\right)} \partial_{2} \mathcal{R}\left(\frac{x_{1}}{\varepsilon}, \frac{y}{\varepsilon}\right) \mathrm{d} y .
$$

Using $\chi \leq 1$ and the upper bound (32) on the profile $\mathcal{R}$, we get the pointwise estimate

$$
\left|\tilde{r}_{\varepsilon}^{d}\left(x_{1}, h\left(x_{1}\right)\right)\right| \leq \int_{0}^{h\left(x_{1}\right)} \frac{C}{1+\left|\frac{x_{1}}{\varepsilon}\right|^{3}} \mathrm{~d} y \leq \frac{C|x|^{4} \varepsilon^{3}}{\varepsilon^{3}+\left|x_{1}\right|^{3}}
$$

which leads to

$$
\int_{\varepsilon}^{r^{*}}\left[\tilde{r}_{\varepsilon}^{d}\left(x_{1}, h\left(x_{1}\right)\right)\right]^{2} \mathrm{~d} x_{1} \leq C \varepsilon^{6} \int_{\varepsilon}^{r^{*}} \frac{\left|x_{1}\right|^{4}}{\left(\varepsilon^{3}+\left|x_{1}\right|^{3}\right)^{2}} \mathrm{~d} x_{1} .
$$

After the change of variables $x_{1}=\varepsilon y$, we finally get

$$
\int_{\varepsilon}^{r^{*}}\left[\tilde{r}_{\varepsilon}^{d}\left(x_{1}, h\left(x_{1}\right)\right)\right]^{2} \mathrm{~d} x_{1} \leq C \varepsilon^{5} \int_{1}^{r^{*} / \varepsilon} \frac{|y|^{4}}{\left(1+|y|^{3}\right)^{2}} \mathrm{~d} y \leq C \varepsilon^{5} .
$$

Let us now address the derivative. For $x=\left(x_{1}, h\left(x_{1}\right)\right) \in \partial \Omega_{\varepsilon} \backslash \varepsilon \partial \omega$, one has

$$
\nabla_{\tau} \tilde{r}_{\varepsilon}^{d}(x)=\chi(x)\left[\partial_{1} \mathcal{R}\left(\frac{x_{1}}{\varepsilon}, \frac{h\left(x_{1}\right)}{\varepsilon}\right)+h^{\prime}\left(x_{1}\right) \partial_{2} \mathcal{R}\left(\frac{x_{1}}{\varepsilon}, \frac{h\left(x_{1}\right)}{\varepsilon}\right)\right]+\nabla_{\tau} \chi(x) \mathcal{R}\left(\frac{x_{1}}{\varepsilon}, \frac{h\left(x_{1}\right)}{\varepsilon}\right) .
$$

We decompose this sum into

$$
\begin{aligned}
& T_{1}(x)=\chi(x) \partial_{1} \mathcal{R}\left(\frac{x_{1}}{\varepsilon}, \frac{h\left(x_{1}\right)}{\varepsilon}\right) \\
& T_{2}(x)=\chi(x) h^{\prime}\left(x_{1}\right) \partial_{2} \mathcal{R}\left(\frac{x_{1}}{\varepsilon}, \frac{h\left(x_{1}\right)}{\varepsilon}\right), \\
& T_{3}(x)=\nabla_{\tau} \chi(x) \mathcal{R}\left(\frac{x_{1}}{\varepsilon}, \frac{h\left(x_{1}\right)}{\varepsilon}\right) .
\end{aligned}
$$

The study of $T_{3}$ is a corollary of (34):

$$
\int_{\varepsilon}^{r^{*}}\left|T_{3}(x)\right|^{2} \mathrm{~d} x_{1} \leq C \varepsilon^{5}
$$

The other terms involve derivation in the fast variable and hence a loss of order. More precisely, we have:

$$
\left|T_{1}(x)\right| \leq\left|\int_{0}^{h\left(x_{1}\right) / \varepsilon} \partial_{2,1}^{2} \mathcal{R}\left(\frac{x_{1}}{\varepsilon}, s\right) \mathrm{d} s\right| \leq \frac{C\left|x_{1}\right|^{2} \varepsilon^{3}}{\varepsilon^{4}+\left|x_{1}\right|^{4}}
$$




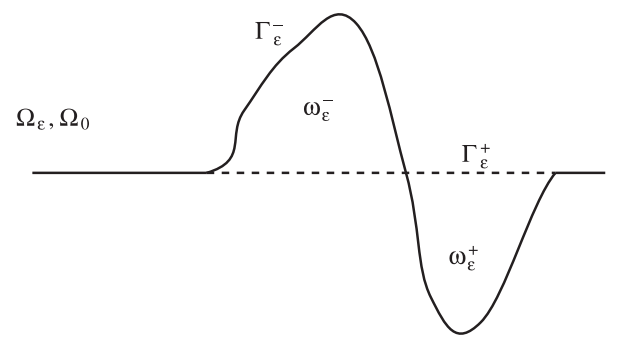

$$
\begin{aligned}
& \Gamma_{\varepsilon}^{-}=\partial \Omega_{\varepsilon} \cap \Omega_{0} \subset \partial \Omega_{\varepsilon}, \\
& \Gamma_{\varepsilon}^{+}=\partial \Omega_{0} \cap \Omega_{\varepsilon} \subset \partial \Omega_{0}, \\
& \omega_{\varepsilon}^{+}=\Omega_{\varepsilon} \backslash\left(\Omega_{\varepsilon} \cap \Omega_{0}\right) \subset \Omega_{\varepsilon}, \\
& \omega_{\varepsilon}^{-}=\Omega_{0} \backslash\left(\Omega_{\varepsilon} \cap \Omega_{0}\right) \subset \Omega_{0} .
\end{aligned}
$$

Figure 4. The notations.

Once we integrate over $x_{1}$, we obtain

$$
\int_{\varepsilon}^{r^{*}}\left|T_{1}\left(x_{1}\right)\right|^{2} \mathrm{~d} x_{1} \leq C \varepsilon^{3} \int_{1}^{r^{*} / \varepsilon} \frac{|y|^{4}}{\left(1+|y|^{3}\right)^{2}} \mathrm{~d} y .
$$

Finally, we write

whence

$$
T_{2}(x) \leq C\left|x_{1}\right| \frac{C}{1+\left|\frac{x_{1}}{\varepsilon}\right|^{3}} \leq \frac{C|x| \varepsilon^{3}}{\varepsilon^{3}+|x|^{3}},
$$

$$
\int_{\varepsilon}^{r^{*}}\left|T_{1}\left(x_{1}\right)\right|^{2} \mathrm{~d} x_{1} \leq C \varepsilon^{3} \int_{1}^{r^{*} / \varepsilon} \frac{|y|^{2}}{\left(1+|y|^{3}\right)^{2}} \mathrm{~d} y .
$$

We treat the double products thanks to the Cauchy-Schwarz inequality to get (35).

All the tools needed to prove the main result of this section are now at our disposal.

Theorem 3.4. In the curved situation described previously, it holds:

$$
u_{\varepsilon}(x)=u_{0}(x)+\chi(x) V_{d}^{1}\left(\frac{x}{\varepsilon}\right)+\mathcal{O}_{\mathrm{H}^{1}\left(\Omega_{\varepsilon}\right)}\left(\varepsilon^{2}\right) .
$$

The boundary condition satisfies

$$
u_{0}(x)+\chi(x) V_{d}^{1}\left(\frac{x}{\varepsilon}\right)=\mathcal{O}_{\mathrm{H}^{1 / 2}\left(\partial \Omega_{\varepsilon}\right)}\left(\varepsilon^{2}\right) .
$$

\section{VARIATIONS OF ENERGY INTEGRALS FOR SINGULAR DOMAIN DEFORMATIONS}

In this section, we investigate the behavior of the Dirichlet energy with respect to singular deformations of the boundary, our presentation is similar to [14]. We recall the definition of the Dirichlet energy of a bounded open subset of $\mathbb{R}^{d}$ : if $f \in \mathcal{D}\left(\mathbb{R}^{d}\right)$ with $\operatorname{supp}(f) \subset \subset \Omega_{0}$,

$$
J\left(\Omega_{0}\right)=-\frac{1}{2} \int_{\Omega_{0}}\left|\nabla u_{\Omega_{0}}(x)\right|^{2} \mathrm{~d} x,
$$

where $u_{\Omega_{0}}$ is the solution of $-\Delta u=f$ in $\mathrm{H}_{0}^{1}\left(\Omega_{0}\right)$. We consider the same class of singular deformations than in the previous section. The notations are recalled in Figure 4. The first result is the following.

Proposition 4.1. Let $\varepsilon>0$ be such that $\operatorname{supp}(f) \subset \subset \Omega_{\varepsilon}$ and $u_{\varepsilon}$ (resp. $u_{0}$ ) denotes the solution of $-\Delta u=f$ in $\mathrm{H}_{0}^{1}\left(\Omega_{\varepsilon}\right)$ (resp. $\mathrm{H}_{0}^{1}\left(\Omega_{0}\right)$ ). Then, one has :

$$
J\left(\Omega_{\varepsilon}\right)=J\left(\Omega_{0}\right)-\frac{1}{2} \int_{\Gamma_{\varepsilon}^{-}} u_{0} \frac{\partial u_{\varepsilon}}{\partial n} \mathrm{~d} \sigma+\frac{1}{2} \int_{\Gamma_{\varepsilon}^{+}} u_{\varepsilon} \frac{\partial u_{0}}{\partial n} \mathrm{~d} \sigma .
$$


Proof of Proposition 4.1. The proof is elementary and based on the Gauss formula. Hence, it can easily be extended to other energy-type functionals (eigenvalues for instance, which express in terms of rayleigh quotients). However, the technique cannot be extended to other shape functionals (e.g. least square fitting to a desire state). Since $\Omega_{\varepsilon}=\left(\Omega_{\varepsilon} \cap \Omega_{0}\right) \cup \omega_{\varepsilon}^{+}$, we write

$$
J\left(\Omega_{\varepsilon}\right)=-\frac{1}{2} \int_{\Omega_{\varepsilon}}\left|\nabla u_{\varepsilon}\right|^{2} \mathrm{~d} x=-\frac{1}{2} \int_{\Omega_{\varepsilon} \cap \Omega_{0}}\left|\nabla u_{0}+\nabla\left(u_{\varepsilon}-u_{0}\right)\right|^{2} \mathrm{~d} x-\frac{1}{2} \int_{\omega_{\varepsilon}^{+}}\left|\nabla u_{\varepsilon}\right|^{2} \mathrm{~d} x .
$$

The second integral can be rewritten via the Gauss formula. One has to be careful with the outer normal vector field. By $\mathbf{n}$, we denote the outer normal vector field of $\partial \Omega_{\varepsilon}$ or $\partial \Omega_{0}$ depending on the context. It may be the opposite to the outer normal field to $\omega_{\varepsilon}^{-}, \omega_{\varepsilon}^{+}$denoted by $n$ :

$$
\int_{\omega_{\varepsilon}^{+}}\left|\nabla u_{\varepsilon}\right|^{2} \mathrm{~d} x=\int_{\omega_{\varepsilon}^{+}} u_{\varepsilon}\left(-\Delta u_{\varepsilon}\right) \mathrm{d} x+\int_{\Gamma_{\varepsilon}^{+}} u_{\varepsilon} \frac{\partial u_{\varepsilon}}{\partial n} \mathrm{~d} \sigma=-\int_{\Gamma_{\varepsilon}^{+}} u_{\varepsilon} \frac{\partial u_{\varepsilon}}{\partial \mathbf{n}} \mathrm{d} \sigma
$$

We expand the first integral and get:

$$
\begin{aligned}
\int_{\Omega_{\varepsilon} \cap \Omega_{0}}\left|\nabla u_{0}+\nabla\left(u_{\varepsilon}-u_{0}\right)\right|^{2} \mathrm{~d} x=\int_{\Omega_{\varepsilon} \cap \Omega_{0}}\left|\nabla u_{0}\right|^{2} \mathrm{~d} x+2 \int_{\Omega_{\varepsilon} \cap \Omega_{0}}\left\langle\nabla u_{0}, \nabla\left(u_{\varepsilon}-u_{0}\right)\right\rangle \mathrm{d} x & +\int_{\Omega_{\varepsilon} \cap \Omega_{0}}\left|\nabla\left(u_{\varepsilon}-u_{0}\right)\right|^{2} \mathrm{~d} x .
\end{aligned}
$$

Applying Green's formula and using the homogeneous Dirichlet boundary conditions on $\partial \Omega_{\varepsilon}$ and $\partial \Omega_{0}$, we have:

$$
\begin{aligned}
\int_{\Omega_{\varepsilon} \cap \Omega_{0}}\left|\nabla u_{0}\right|^{2} \mathrm{~d} x & =\int_{\Omega}\left|\nabla u_{0}\right|^{2} \mathrm{~d} x+\int_{\Gamma_{\varepsilon}^{-}} u_{0} \frac{\partial u_{0}}{\partial \mathbf{n}} \mathrm{d} \sigma \\
\int_{\Omega_{\varepsilon} \cap \Omega_{0}}\left|\nabla\left(u_{\varepsilon}-u_{0}\right)\right|^{2} \mathrm{~d} x & =\int_{\Gamma_{\varepsilon}^{-}} u_{0}\left(\frac{\partial u_{0}}{\partial \mathbf{n}}-\frac{\partial u_{\varepsilon}}{\partial \mathbf{n}}\right) \mathrm{d} \sigma+\int_{\Gamma_{\varepsilon}^{+}} u_{\varepsilon}\left(\frac{\partial u_{\varepsilon}}{\partial \mathbf{n}}-\frac{\partial u_{0}}{\partial \mathbf{n}}\right) \mathrm{d} \sigma \\
\int_{\Omega_{\varepsilon} \cap \Omega_{0}}\left\langle\nabla u_{0}, \nabla\left(u_{\varepsilon}-u_{0}\right)\right\rangle \mathrm{d} x & =\int_{\Gamma_{\varepsilon}^{-}} u_{0} \frac{\partial u_{\varepsilon}}{\partial \mathbf{n}} \mathrm{d} \sigma-\int_{\Gamma_{\varepsilon}^{-}} u_{0} \frac{\partial u_{0}}{\partial \mathbf{n}} \mathrm{d} \sigma .
\end{aligned}
$$

We now sum up all these intermediary computations, and we get:

$$
\int_{\Omega_{\varepsilon} \cap \Omega_{0}}\left|\nabla u_{\varepsilon}\right|^{2} \mathrm{~d} x=\int_{\Omega_{0}}\left|\nabla u_{0}\right|^{2} \mathrm{~d} x+\int_{\Gamma_{\varepsilon}^{-}} u_{0} \frac{\partial u_{\varepsilon}}{\partial \mathbf{n}} \mathrm{d} \sigma+\int_{\Gamma_{\varepsilon}^{+}} u_{\varepsilon}\left(\frac{\partial u_{\varepsilon}}{\partial \mathbf{n}}-\frac{\partial u_{0}}{\partial \mathbf{n}}\right) \mathrm{d} \sigma
$$

and

This concludes the proof.

$$
\int_{\Omega_{\varepsilon}}\left|\nabla u_{\varepsilon}\right|^{2} \mathrm{~d} x=\int_{\Omega_{0}}\left|\nabla u_{0}\right|^{2} \mathrm{~d} x+\int_{\Gamma_{\varepsilon}^{-}} u_{0} \frac{\partial u_{\varepsilon}}{\partial \mathbf{n}} \mathrm{d} \sigma-\int_{\Gamma_{\varepsilon}^{+}} u_{\varepsilon} \frac{\partial u_{0}}{\partial \mathbf{n}} \mathrm{d} \sigma .
$$

Change of boundary conditions. The same method allows to handle other boundary conditions on the perturbed part of the boundary. Assume that the state function $u_{\Omega_{\varepsilon}}$ now solves the mixed problem

$$
\left\{\begin{aligned}
-\Delta u & =f \in \Omega_{\varepsilon}, \\
u & =0 \in \partial \Omega_{\varepsilon} \cap \partial \Omega_{0}, \\
\partial_{n} u & =0 \in \partial \Omega_{\varepsilon} \backslash\left(\partial \Omega_{\varepsilon} \cap \partial \Omega_{0}\right) .
\end{aligned}\right.
$$

We can state the counterpart of Proposition 4.1. 
Proposition 4.2. Let $\varepsilon>0$ be such that $\operatorname{supp}(f) \subset \subset \Omega_{\varepsilon}$ and $u_{\varepsilon}$ denotes the solution of $(39)$ in $\mathrm{H}^{1}\left(\Omega_{\varepsilon}\right)$. Let $u_{0}$ be the solution of $-\Delta u=f$ in $\left.\mathrm{H}_{0}^{1}\left(\Omega_{0}\right)\right)$. Then, one has:

$$
J\left(\Omega_{\varepsilon}\right)=J\left(\Omega_{0}\right)+\frac{1}{2} \int_{\Gamma_{\varepsilon}^{-}} u_{0} \frac{\partial u_{0}}{\partial n} \mathrm{~d} \sigma-\frac{1}{2} \int_{\Gamma_{\varepsilon}^{+}} u_{\varepsilon} \frac{\partial u_{\varepsilon}}{\partial n} \mathrm{~d} \sigma .
$$

The proof is very similar to the proof of Proposition 4.1. The changes appear in the Green formula.

Inserting the asymptotic expansion of $u_{\varepsilon}$ into formulæ (38) and (40), we easily obtain

Proposition 4.3. In the framework of Proposition 4.1, the Dirichlet energy admits the following asymptotic expansion:

$$
J\left(\Omega_{\varepsilon}\right)=J\left(\Omega_{0}\right)+\varepsilon^{2}\left|\nabla u_{0}(0)\right|^{2} \mathcal{A}_{\mathbf{H}_{\infty}}+\mathcal{o}\left(\varepsilon^{2}\right),
$$

where the number $\mathcal{A}_{\mathbf{H}_{\infty}}$ called polarisation number by analogy with the polarisation matrix of Polya writes

$$
\mathcal{A}_{\mathbf{H}_{\infty}}=-\frac{1}{2} \int_{\Gamma^{-}} K(y) \partial_{N} K(y) \mathrm{d} \sigma_{y}+\frac{1}{2} \int_{\Gamma^{+}} K(y) N_{2}(y) \mathrm{d} \sigma_{y}
$$

$K$ is the normalized profile: $K=V_{d}^{1} /\left|\nabla u_{0}(0)\right|$, cf. (10).

Proposition 4.4. In the framework of Proposition 4.2, formula (41) hold with the modified polarisation number

$$
\mathcal{A}_{\mathbf{H}_{\infty}}=-\frac{1}{2} \int_{\Gamma^{-}} N_{2}(y) \mathrm{d} \sigma_{y}+\frac{1}{2} \int_{\Gamma^{+}} K(y) \partial_{N} K(y) \mathrm{d} \sigma_{y}
$$

with the modified boundary conditions.

\section{Numerics}

\subsection{Strategy}

As already mentioned, the solution $u_{\varepsilon}$ of the model problem (5) is difficult to approximate from a numerical point of view: the refinement needed near the perturbation for a reasonable precision prevents (at least for small values of $\varepsilon$ ) to compute $u_{\varepsilon}$ directly. The asymptotic expansion, see Theorem 2.2 , suggests the following numerical strategy.

Writing the expansion $(24)$ of $u_{\varepsilon}$ at order 1 , we get

$$
u_{\varepsilon}(x) \simeq \zeta\left(\frac{x}{\varepsilon}\right) u_{0}(x)+\varepsilon \chi(x) V^{1}\left(\frac{x}{\varepsilon}\right) .
$$

For simplicity, we consider here the case of a cavity $\left(\Omega_{\varepsilon} \subset \Omega_{0}\right)$ and thanks to Remark 2.3, the cut-off function $\zeta$ may be chosen identically equal to 1 . A natural approximation of $u_{\varepsilon}$ reads then $u_{\varepsilon}(x) \simeq u_{0}(x)+\varepsilon \chi(x) V^{1}\left(\frac{x}{\varepsilon}\right)$.

- The limit term $u_{0}$ may be computed accurately in a pretty coarse mesh independently of $\varepsilon$;

- the profile $V^{1}$ does not depend on $\varepsilon$, but only on the geometry of the pattern $\mathbf{H}_{\infty}$. Its approximation is not straightforward, since it is defined on an unbounded domain. Various techniques are available for the numerical resolution of such a problem in an unbounded domain with an unbounded boundary. The basic idea consists in bounding the domain by an artificial boundary $\Sigma$ on which we impose a suitable boundary condition. This condition should take into account the behavior at infinity $V^{1}(X) \rightarrow 0$ as $|X| \rightarrow 0$.

(i) Simplest choice: impose a homogeneous Dirichlet condition $V=0$ on $\Sigma=B(0, R) \cup \partial \mathbf{H}_{\infty}$. In this case, the radius $R$ of the ball has to be large enough. Depending on the target precision, this naive - but easy to implement - solution may be acceptable. 
(ii) Use the knowledge concerning the behavior at infinity of the profile $V^{1}$ : rather than 0 , we impose the condition $V=R^{-1} \sin \theta$ on $\Sigma$, i.e. the leading term in the expansion at infinity of the profile $V^{1}$. The results are much better and we used this method in the the model computations shown in next section.

(iii) Numerous more accurate artificial boundary conditions are available, most of them developed in the framework of wave propagation: local transparent conditions (see $[6,7]$ ) or non-local "exactly absorbing" conditions using an integral representation (localized finite element method [11]).

The functions $u_{0}$ and $V^{1}$ being computed, it remains to perform the superposition of $u_{0}(x)$ with the correcting term $\varepsilon \chi(x) V^{1}\left(\frac{x}{\varepsilon}\right)$. Since the mesh used for the approximations do not coincide, we need to transfer $V^{1}(\dot{\bar{\varepsilon}})$ onto the mesh where $u_{0}$ has been computed. This step can be facilitated by using a regular mesh for $V^{1}$ (e.g. cartesian in polar coordinates, except near the perturbing pattern). The function $\chi$ is replaced in the computations by the use of a patch of elements: $V^{1}$ is not taken into account except in this patch.

The obtained approximation is close to $u_{\varepsilon}$ up to order $\mathcal{O}\left(\varepsilon^{2}\right)$. For small values of $\varepsilon$, we expect the method to work fine; for larger $\varepsilon$, the results may be inaccurate, but in that case the perturbation can be incorporated directly to the initial mesh without harsh refinement. Of course, from a practical point of view small and large have to be adapted to the considered situation.

\subsection{Numerical results}

We present some numerical results which validate our approach. The considered problem is the following

$$
u_{\varepsilon} \in \mathrm{H}^{1}\left(\Omega_{\varepsilon}\right),-\Delta u_{\varepsilon}=f \text { in } \Omega_{\varepsilon} \text {, and } \partial_{n} u=0 \text { on } \partial \Omega_{\varepsilon},
$$

where $f(x, y)=2 \pi^{2} \cos (\pi x) \sin (\pi y)$ and $\Omega_{\varepsilon}$ is the square $(-1 / 2,1 / 2) \times(0,1)$ with a semicircular hole of radius $\varepsilon$, centered at $(0,0)$. Figure 5 and Table 1 show, for $\varepsilon=1 / 32$ the solution $u_{\varepsilon}$ (top-left picture), the difference between $u_{\varepsilon}$ and the limit term $u_{0}$ (top-right picture), the difference between $u_{\varepsilon}$ and the corrected limit term $u_{1}=u_{0}+\varepsilon V^{1}(\dot{\dot{\varepsilon}})$ (bottom-right picture ${ }^{2}$ ). The bottom-left graph represents, for various values of $\varepsilon$, the norm of the errors $\left(\mathrm{L}^{2}, \mathrm{H}^{1}\right.$ and $\mathrm{L}^{\infty}$-norms).

The efficiency of the correction by the first profile clearly appears in these results: for example, with $\varepsilon=1 / 128$, the $\mathrm{L}^{\infty}$-norm of $u_{\varepsilon}-u_{1}$ is about 40 times less than the $\mathrm{L}^{\infty}$-norm of $u_{\varepsilon}-u_{0}$.

In Figure 6 and Table 2 , we present the same results in the Dirichlet case for a curved $\Omega_{0}$ :

$$
u_{\varepsilon} \in \mathrm{H}^{1}\left(\Omega_{\varepsilon}\right),-\Delta u_{\varepsilon}=f \text { in } \Omega_{\varepsilon} .
$$

The same conclusions arise; the gain in $\mathrm{L}^{\infty}$-norm is here around 50.

\section{EXTENSION TO LINEAR ELASTICITY AND POSSIBLE APPLICATION TO MECHANICAL ENGINEERING}

It turns out that the presented results naturally extend to linear elasticity. Indeed the technique relies on the construction of the profiles, which requires essentially a variational framework for the unbounded problem. Of course as counterpart of one profile for the Laplace equation, two profiles have to be introduced here, since the unknown is a two-dimensional vector. Precisely, writing the equations in the Naviers form:

$$
\left\{\begin{array}{l}
-\mu \Delta \mathbf{u}_{\varepsilon}-(\lambda+\mu) \operatorname{grad} \operatorname{div} \mathbf{u}_{\varepsilon}=\mathbf{f} \text { in } \Omega_{\varepsilon} \\
\mathbf{u}_{\varepsilon}=\mathbf{0} \text { on } \Gamma_{D} \\
\sum_{j=1}^{2} \sigma_{i j}\left(\mathbf{u}_{\varepsilon}\right) \mathbf{n}_{j}=\mathbf{g}_{i} \text { on } \hat{\mathrm{E}} \partial \Omega_{\varepsilon} \backslash \Gamma_{D}
\end{array}\right.
$$

\footnotetext{
${ }^{2}$ The profile $V^{1}$ has been computed on a (quasi-)regular mesh, independently of the value of $\varepsilon$, and it has been projected onto the initial grid for the computation of $u_{1}$.
} 


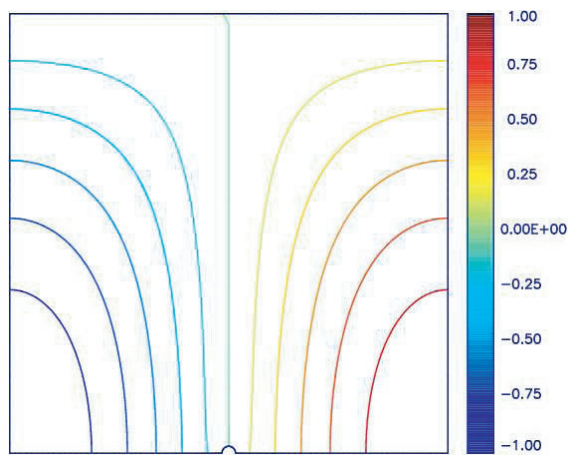

$u_{\varepsilon}$ for $\varepsilon=1 / 32$

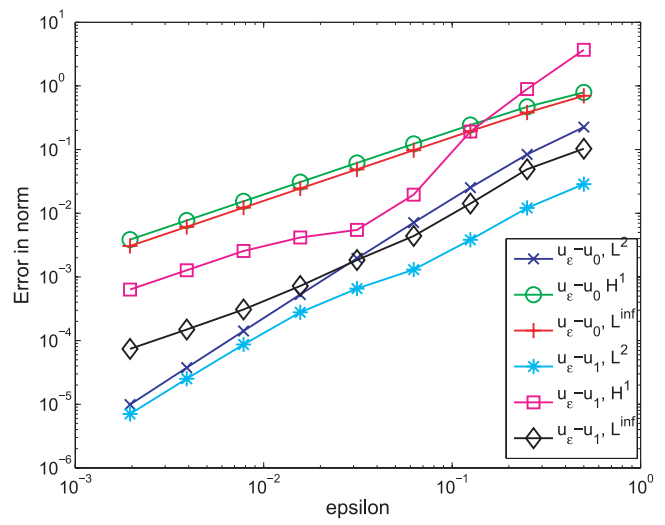

Norms of the errors

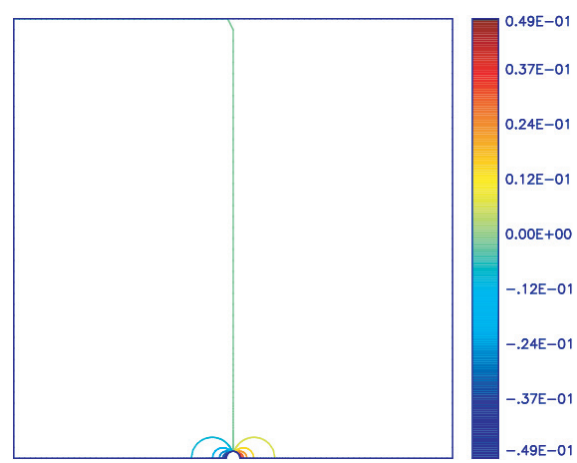

$u_{\varepsilon}-u_{0}$ for $\varepsilon=1 / 32$

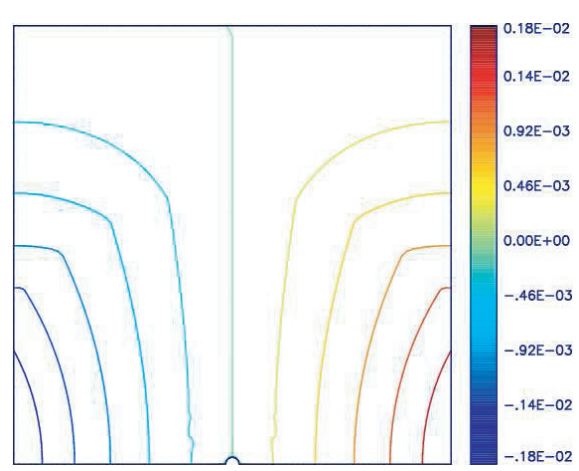

$u_{\varepsilon}-u_{1}$ for $\varepsilon=1 / 32$

Figure 5. Computations for the Neumann problem (43).

TABle 1. Computations for the Neumann problem (43).

\begin{tabular}{|c|c|c|c|}
\hline$\varepsilon$ & $\left\|u_{\varepsilon}-u_{0}\right\|_{\mathrm{H}^{1}\left(\Omega_{\varepsilon}\right)}$ & $\left\|u_{\varepsilon}-u_{1}\right\|_{\mathrm{H}^{1}\left(\Omega_{\varepsilon}\right)}$ & Gain \\
\hline$\varepsilon=1 / 2$ & $7.9168 \mathrm{e}-01$ & $3.6946 \mathrm{e}+00$ & 0.2 \\
\hline$\varepsilon=1 / 4$ & $4.6937 \mathrm{e}-01$ & $8.9397 \mathrm{e}-01$ & 0.5 \\
\hline$\varepsilon=1 / 8$ & $2.4354 \mathrm{e}-01$ & $1.9181 \mathrm{e}-01$ & 1.3 \\
\hline$\varepsilon=1 / 16$ & $1.2286 \mathrm{e}-01$ & $1.9593 \mathrm{e}-02$ & 6.3 \\
\hline$\varepsilon=1 / 32$ & $6.1569 \mathrm{e}-02$ & $5.4704 \mathrm{e}-03$ & 11.3 \\
\hline$\varepsilon=1 / 64$ & $3.0802 \mathrm{e}-02$ & $4.1649 \mathrm{e}-03$ & 7.4 \\
\hline$\varepsilon=1 / 128$ & $1.5403 \mathrm{e}-02$ & $2.5574 \mathrm{e}-03$ & 6.0 \\
\hline$\varepsilon=1 / 256$ & $7.7017 \mathrm{e}-03$ & $1.2696 \mathrm{e}-03$ & 6.1 \\
\hline$\varepsilon=1 / 512$ & $3.8509 \mathrm{e}-03$ & $6.3292 \mathrm{e}-04$ & 6.1 \\
\hline
\end{tabular}

with $\mathbf{f}$ and $\mathbf{g}_{i}$ vanish near the perturbation, we have the first order formula (in the inclusive case, for simplicity)

$$
\mathbf{u}_{\varepsilon}(x) \simeq \mathbf{u}_{1}(x) \stackrel{\text { def. }}{=} \mathbf{u}_{0}(x)+\varepsilon \chi(x)\left[\alpha_{1} \mathbf{V}_{1}^{1}\left(\frac{x}{\varepsilon}\right)+\alpha_{2} \mathbf{V}_{2}^{1}\left(\frac{x}{\varepsilon}\right)\right]
$$




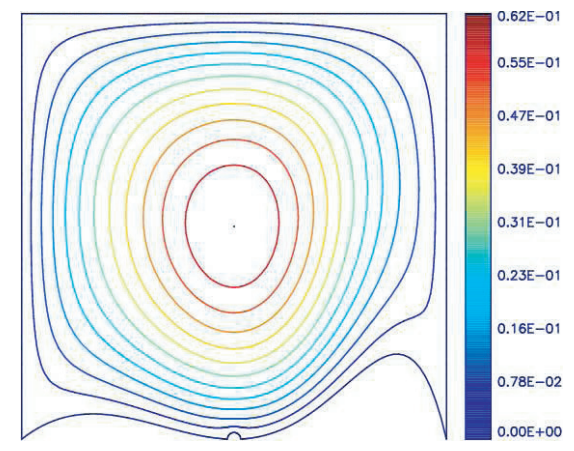

$u_{\varepsilon}$ for $\varepsilon=1 / 32$

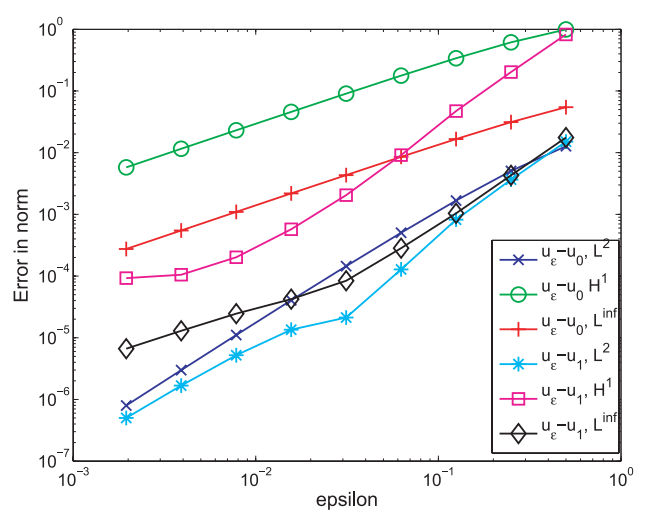

Norms of the errors

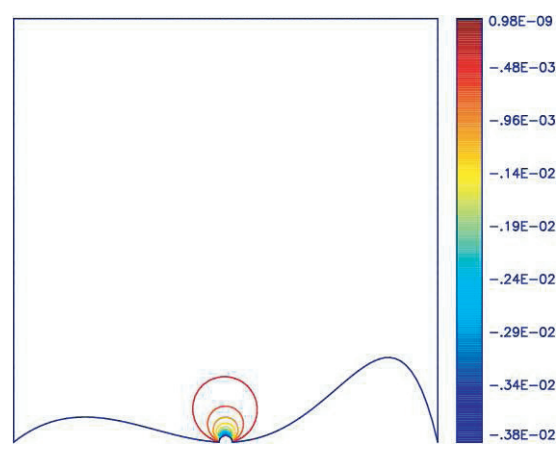

$u_{\varepsilon}-u_{0}$ for $\varepsilon=1 / 32$

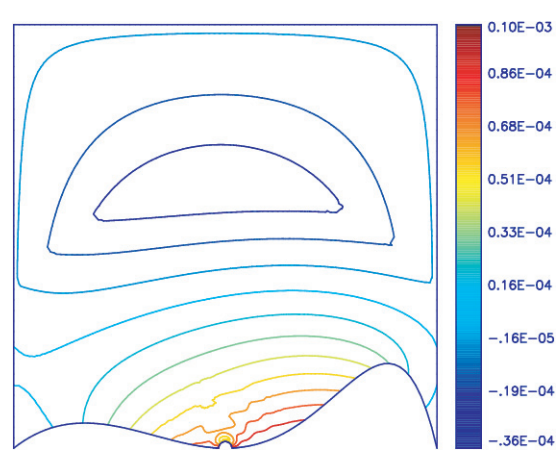

$u_{\varepsilon}-u_{1}$ for $\varepsilon=1 / 32$

Figure 6. Computations for the Dirichlet problem (44).

TABLE 2. Computations for the Dirichlet problem (44).

\begin{tabular}{|c|c|c|c|}
\hline$\varepsilon$ & $\left\|u_{\varepsilon}-u_{0}\right\|_{\mathrm{H}^{1}\left(\Omega_{\varepsilon}\right)}$ & $\left\|u_{\varepsilon}-u_{1}\right\|_{\mathrm{H}^{1}\left(\Omega_{\varepsilon}\right)}$ & Gain \\
\hline$\varepsilon=1 / 2$ & $9.9264 \mathrm{e}-01$ & $8.2636 \mathrm{e}-01$ & 1.2 \\
\hline$\varepsilon=1 / 4$ & $6.1395 \mathrm{e}-01$ & $2.0264 \mathrm{e}-01$ & 3.0 \\
\hline$\varepsilon=1 / 8$ & $3.3907 \mathrm{e}-01$ & $4.7275 \mathrm{e}-02$ & 7.2 \\
\hline$\varepsilon=1 / 16$ & $1.7746 \mathrm{e}-01$ & $9.1492 \mathrm{e}-03$ & 19.4 \\
\hline$\varepsilon=1 / 32$ & $9.0685 \mathrm{e}-02$ & $2.0483 \mathrm{e}-03$ & 44.3 \\
\hline$\varepsilon=1 / 64$ & $4.5827 \mathrm{e}-02$ & $5.7374 \mathrm{e}-04$ & 79.9 \\
\hline$\varepsilon=1 / 128$ & $2.3034 \mathrm{e}-02$ & $2.0183 \mathrm{e}-04$ & 114.1 \\
\hline$\varepsilon=1 / 256$ & $1.1547 \mathrm{e}-02$ & $1.0518 \mathrm{e}-04$ & 109.8 \\
\hline$\varepsilon=1 / 512$ & $5.7810 \mathrm{e}-03$ & $9.2734 \mathrm{e}-05$ & 62.3 \\
\hline
\end{tabular}

- The profiles $\mathbf{V}_{\ell}^{1}(\ell=1,2)$ solve the homogeneous problem

$$
-\mu \Delta \mathbf{V}_{\ell}^{1}-(\lambda+\mu) \operatorname{grad} \operatorname{div} \mathbf{V}_{\ell}^{1}=\mathbf{0} \text { in } \mathbf{H}_{\infty} \quad \text { and } \sum_{j=1}^{2} \sigma_{i j}\left(\mathbf{V}_{\ell}^{1}\right) \mathbf{n}_{j}=\mathbf{G}_{\ell, i} \text { on } \partial \mathbf{H}_{\infty}
$$

with $\mathbf{G}_{1}=\left(\mathbf{N}_{1}, 0\right)$ and $\mathbf{G}_{2}=\left(0, \mathbf{N}_{1}\right)\left(\mathbf{N}_{1}\right.$ is the first component of the outer normal on $\left.\partial \mathbf{H}_{\infty}\right)$. 


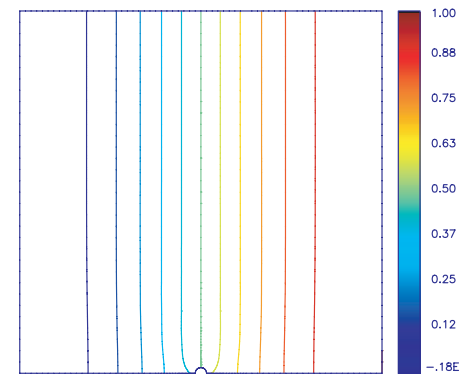

$\left(\mathrm{u}_{\varepsilon}\right)_{x}$ for $\varepsilon=1 / 32$

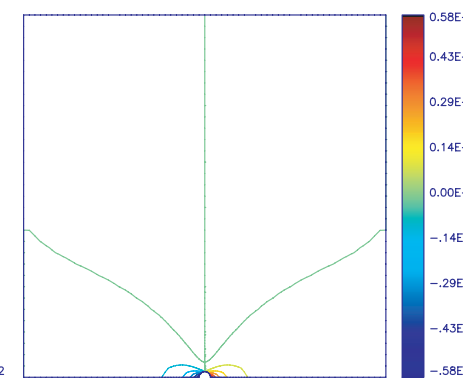

$\left(\mathrm{u}_{\varepsilon}-\mathrm{u}_{0}\right)_{x}$ for $\varepsilon=1 / 32$

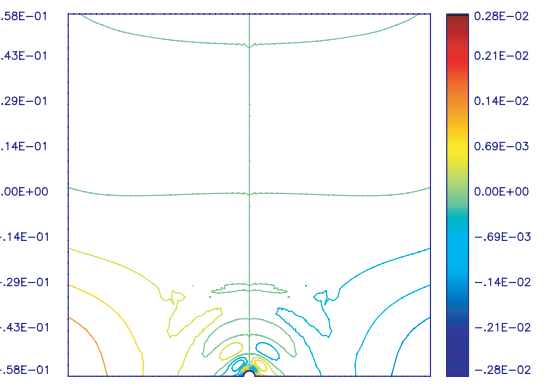

$\left(\mathrm{u}_{\varepsilon}-\mathrm{u}_{1}\right)_{x}$ for $\varepsilon=1 / 32$

Figure 7. Computations for linear elasticity (45).

- The coefficient $\alpha_{\ell}$ is given by $\alpha_{\ell}=-\sigma_{\ell 1}\left(\mathbf{u}_{0}\right)$.

The presented techniques have applications in civil engineering. With colleagues from computational mechanics, we are interested in investigating the influence of surface defects on the initiation of a crack and its propagation in a material (typically concrete). The goal is to predict where the material should break. Basically, the mechanical behavior splits into three phases: a first elastic one before the crack initiation, next the apparition of microcracks, and last the development of macrocracks responsible for the rupture. We propose to treat the first step with the tools of multiscale correction, to predict where the first cracks will occur. The superposition technique is based on the linearity of the operator. Hence it is justified only in the first phase. We handle the second and third phases with a continuum damage model and a strong discontinuity approach (see[2]).

The superposition technique has been inserted into the main code for crack initiation. The numerical tests are being carried out and first results have been presented in the conference [3] without proceedings, a paper is under redaction. We present in Figure 7 the first component of the solution $\mathbf{u}_{\varepsilon}$ and of the errors $\mathbf{u}_{\varepsilon}-\mathbf{u}_{0}$ and $\mathbf{u}_{\varepsilon}-\mathbf{u}_{1}$ for the value $\varepsilon=1 / 32$. The data for the computation are the following: $\lambda=0.5769,2 \mu=0.7692$ and $\mathbf{f}$ corresponds to $\mathbf{u}_{0}=[1 / 2+1 / 2 \sin (\pi x),(-\lambda \cos (\pi x) \sin (2 \pi y)) /(\lambda+2 \mu)]$; the boundary conditions are homogeneous Neumann on the top and bottom sides, $\mathbf{u}_{\varepsilon}=\mathbf{0}$ on the left side and $\mathbf{u}_{\varepsilon}=(1,0)$ on the right side.

Of course, we would like to take into account the surface defects in the second phase as well, but the description is no more linear. It is very challenging to extend our ideas to this situation which involves a damaged phase and hence nonlinearities. Such an extension is out of reach with the methods presented in this work.

\section{REFERENCES}

[1] G. Allaire, Shape optimization by the homogenization method, Applied Mathematical Sciences 146. Springer-Verlag, New York (2002).

[2] D. Brancherie and A. Ibrahimbegović, Modélisation 'macro' de phénomènes localisés à l'échelle 'micro' : formulation et implantation numérique. Revue européenne des éléments finis, numéro spécial Giens 200313 (2004) 461-473.

[3] D. Brancherie, M. Dambrine, G. Vial and P. Villon, Ultimate load computation, effect of surfacic defect and adaptative techniques, in 7th World Congress in Computational Mechanics, Los Angeles (2006).

[4] G. Caloz, M. Costabel, M. Dauge and G. Vial, Asymptotic expansion of the solution of an interface problem in a polygonal domain with thin layer. Asymptotic Anal. 50 (2006) 121-173.

[5] M. Dambrine and G. Vial, On the influence of a boundary perforation on the dirichlet energy. Control Cybern. 34 (2005) $117-136$.

[6] B. Engquist and A. Majda, Absorbing boundary conditions for the numerical simulation of waves. Math. Comp. 31 (1977) 629-651.

[7] D. Givoli, Nonreflecting boundary conditions. J. Comput. Phys. 94 (1991) 1-29.

[8] A.M. Il'lin, Matching of Asymptotic Expansions of Solutions of Boundary Value Problems. Translations of Mathematical Monographs 102, Amer. Math. Soc., Providence, R.I. (1992).

[9] V.A. Kondrat'ev, Boundary value problems for elliptic equations in domains with conical or angular points. Trans. Moscow Math. Soc. 16 (1967) 227-313. 
[10] D. Leguillon and E. Sanchez-Palencia, Computation of singular solutions in elliptic problems and elasticity. Masson, Paris (1987).

[11] M. Lenoir and A. Tounsi, The localized finite element method and its application to the two-dimensional sea-keeping problem. SIAM J. Numer. Anal. 25 (1988) 729-752.

[12] T. Lewiński and J. Sokołowski, Topological derivative for nucleation of non-circular voids. The Neumann problem, in Differential geometric methods in the control of partial differential equations (Boulder, CO, 1999), Contemp. Math. 268, Amer. Math. Soc., Providence, RI (2000) 341-361.

[13] M. Masmoudi, The Topological Asymptotic, in Computational Methods for Control Applications, International Séries GAKUTO (2002)

[14] V.G. Maz'ya and S.A. Nazarov, Asymptotic behavior of energy integrals under small perturbations of the boundary near corner and conic points. Trudy Moskov. Mat. Obshch. 50 (1987) 79-129, 261.

[15] V.G. Maz'ya, S.A. Nazarov and B.A. Plamenevskij, Asymptotic theory of elliptic boundary value problems in singularly perturbed domains. Birkhäuser, Berlin (2000).

[16] S.A. Nazarov and M.V. Olyushin, Perturbation of the eigenvalues of the Neumann problem due to the variation of the domain boundary. Algebra i Analiz 5 (1993) 169-188.

[17] S.A. Nazarov and J. Sokołowski, Asymptotic analysis of shape functionals. J. Math. Pures Appl. 82 (2003) 125-196.

[18] S. Tordeux and G. Vial, Matching of asymptotic expansions and multiscale expansion for the rounded corner problem. $S A M$ Research Report, ETH, Zürich (2006). 